\title{
Mean grain diameters from thin sections: matching the average to the problem
}

\author{
Robert S. FArR ${ }^{1, *}$, Victoria C. Honour ${ }^{2}$ and Marian B. Holness ${ }^{2}$ \\ 1 Unilever RD Colworth Science Park, Sharnbrook, Bedford MK44 1LQ, UK \\ 2 Department of Earth Sciences, University of Cambridge, Downing Street, Cambridge CB2 3EQ, UK
}

[Received 4 November 2015; Accepted 12 April 2016; Associate Editor: Brian O’Driscoll]

\section{ABSTRACT}

It is common practice to estimate a mean diameter for spherical or sub-spherical particles or vesicles in a rock by multiplying the average diameter of the approximately circular cross-sections visible in thin section by a factor of 1.273 . This number-weighted average may be dominated by the hard-to-measure fine tail of the size distribution, and is unlikely to be representative of the average particle diameter of greatest interest for a wide range of geological problems or processes. Average particle size can be quantified in a variety of ways, based on the mass or surface area of the particles, and here we provide exact relations of these different average measures to straightforward measurements possible in thin section, including an analysis of how many particles to measure to achieve a desired level of uncertainty. The use of average particle diameter is illustrated firstly with a consideration of the accumulation of olivine phenocrysts on the floor of the $135 \mathrm{~m}$ thick picrodolerite/crinanite unit of the Shiant Isles Main Sill. We show that the $45 \mathrm{~m}$ thick crystal pile on the sill floor could have formed by crystal settling within about a year. The second geological example is provided by an analysis of the sizes of exsolved Fe-rich droplets during unmixing of a basaltic melt in a suite of experimental charges. We show that the size distribution cannot be explained by sudden nucleation, followed by either Ostwald ripening or Brownian coalescence. We deduce that a continuous process of droplet nucleation during cooling is likely to have occurred.

KEYWORDs: petrology, microstructure, grain size, crystal settling, liquid immiscibility.

\section{Introduction}

USEFUL answers to many geological problems can be obtained from relatively simple calculations that provide time- or length-scales, correct within an order of magnitude, which can then be used to place constraints on the processes likely to have been involved in the problem in question. Good examples of this kind of approach are based on determinations of grain size, with the quantification of particle size in rocks (either grains or bubbles) providing an opportunity to make progress on many problems of petrological interest, such as magma solidification time-scales (Cashman and Marsh, 1988; Cashman, 1993; Higgins, 1996),

*E-mail: robert.s.farr@gmail.com

https://doi.org/10.1180/minmag.2016.080.107 crystallization during magma ascent (Cashman, 1992; Hammer et al., 1999), rates of production and coalescence of volatile-filled bubbles from magma (Herd and Pinkerton, 1997), buoyancy-driven particle migration or other fluid dynamical processes (Robertson and Barnes, 2015), rates of Ostwald ripening (Cabane et al., 2001, 2005) and pattern formation in metamorphic rocks (Holness, 1997).

Much recent work using grain size to quantify geological processes is based on a sophisticated treatment involving the characterization and interpretation of the particle size distribution (as introduced by Marsh, 1988). The accuracy of such an approach is enhanced by determination of the true 3D distribution of grain sizes by disaggregation, dissolution of the matrix (e.g. Holness, 1997) or tomographic analysis (e.g. Carlson and Denison, 1992; Denison and Carlson, 1997). However, given 
the limitations of the materials we work with, most studies using grain size are based on observations of thin sections, in which case stereological corrections are required to convert the range of grain intersection size to a 3D grain-size distribution (e.g. Cashman and Marsh, 1988; Johnson, 1994; Higgins, 2000).

For spherical particles, converting the distribution of circular cross-sections observed in thin section into an estimate for the true 3D distribution of particle diameters is mathematically well defined (Wicksell, 1925). For non-spherical particles, such as parallelepipeds, available numerical methods are based on the assumption of invariant particle shape regardless of size (e.g. Higgins, 1994; Sahagian and Proussevitch, 1998; Morgan and Jerram, 2006), which is not likely to be true for natural samples (e.g. Mock and Jerram, 2005; Duchêne et al., 2008). However, for many applications a mean particle diameter is often sufficient to provide order of magnitude estimates that can be used to constrain timescales of geologically interesting problems. The question then arises as to how one might obtain a mean particle diameter from thin-section observations.

For the particular case of a monodisperse population of spheres (i.e. one with a uniform particle size), the average diameter of circular crosssections obtained by random cross-sections through the population is $\pi / 4$ times the sphere diameter. The simplicity of this relationship has led to its common application to estimate an average 3D particle diameter for polydisperse (i.e. a population with a range of 3D particle sizes) as well as monodisperse particle distributions (Hughes, 1978; Cashman and Marsh, 1988; Kong et al., 1995; Herd and Pinkerton, 1997), although model-based maximum likelihood approaches are also used (Kong et al., 1995).

In this contribution we concentrate on systems containing spherical particles, such as bubbles, droplets in an emulsion, or equant mineral grains such as olivine or spinel, and argue that such a simple approach to determining the average particle diameter has three problems, which can be remedied easily. Firstly, the average value obtained using this method may be affected strongly by the smallest particles in the population, which is precisely the part of the size distribution that is most likely to be overlooked or not properly resolved. Secondly, this approach provides no estimate of the uncertainties in the result. Thirdly, and perhaps most importantly, it doesn't address a question of great significance for polydisperse particle populations which is absent for the monodisperse case, namely: which of the various ways of calculating the average diameter is most appropriate for the problem we are interested in?

Firstly we discuss the various merits of different ways of calculating the average for sphere diameters, present some simple exact results linking them to circular cross-sections, and provide simulated data to show how many grains need to be measured to achieve any required degree of accuracy. We then explore how sensitive these statistics are to ignoring the smallest cross-sections in a sample, and whether the proposed method can be applied to non-spherical, but equant grains (specifically, we look at cubes). Lastly we illustrate the usefulness of various measures of the average particle size to constrain timescales of settling of olivine grains on the floor of a basaltic sill, and the mechanisms of coarsening of an unmixed immiscible basaltic melt.

\section{Calculating the average}

Let us consider a collection of $v_{\mathrm{sph}}$ spherical particles with diameters $\left\{D_{i}\right\}$, where $1 \leq i \leq v_{\mathrm{sph}}$. One way to characterize this distribution is to find the 'number-weighted' mean diameter which we will term $D_{1,0}$. This is simply the sum of all the sphere diameters divided by the number of spheres:

$$
D_{1,0} \equiv \frac{1}{v_{\mathrm{sph}}} \sum_{i=1}^{v_{\mathrm{sph}}} D_{i}=\sum_{i} D_{i} / \sum_{i} D_{i}^{0} .
$$

However, this simple average may be problematic, particularly if the grain size distribution is broad, as an abundance of very small grains will dominate this average so that $D_{1,0}$ may be much smaller than we expect. Additionally, it may be difficult to identify all the tiniest particles (for example, within the limits of the X-ray tomographic analysis of any particular sample), so that even if we were happy to use $D_{1,0}$ the calculated value might be prone to large errors.

There are in fact many other ways to take an average diameter. For a collection of spheres of diameters $\left\{D_{i}\right\}$, we define the moment-based average $D_{j, k}$ by

$D_{j, k} \equiv\left\{\begin{array}{cc}{\left[\sum_{i} D_{i}^{j} / \sum_{i} D_{i}^{k}\right]^{1 /(j-k)}} & j \neq k \\ \exp \left[\sum_{i} \ln \left(D_{i}\right) D_{i}^{j} / \sum_{i} D_{i}^{j}\right] & j=k\end{array}\right.$

The case $j=k$ comes simply from taking the limit $j \rightarrow k$ in the first line of equation 2 . We note as an aside that in general $D_{k+1, k} \geq D_{k, k-1}$ (with equality only when the system is monodisperse), and also (Alderliesten, 1990) that $D_{j, k}=D_{k, j}$. 
For problems of geological interest perhaps the most useful of these averages are the volumeweighted mean diameter $D_{4,3}$ and the surfaceweighted mean diameter $D_{3,2}$ :

$$
\begin{gathered}
D_{4,3} \equiv \sum_{i} D_{i}^{4} / \sum_{i} D_{i}^{3}, \\
D_{3,2} \equiv \sum_{i} D_{i}^{3} / \sum_{i} D_{i}^{2} .
\end{gathered}
$$

The volume-weighted mean diameter can be defined as follows: choose random points in space, and if the point lies within one of the particles of interest, record the diameter of that particle. $D_{4,3}$ is the mean of all the diameters sampled this way. Because large spheres are much more likely to be sampled than small spheres, this average is dominated by the larger spheres in the distribution, and much less sensitive to underrepresentation of the tail of fine dust. The areaweighted mean diameter $D_{3,2}$ can be thought of as taking a mean of diameters where spheres in the sample are chosen with a probability proportional to their surface area (rather than volume as in $D_{4,3}$ ). It also is less sensitive to the tail of fine particles than the simple number-weighted average.

The choice of average diameter depends on which captures the relevant properties of the system under investigation. $D_{4,3}$ represents the size class around which most of the mass of the particles lies, and for that reason may be taken as a good measure of particle diameter from a compositional point of view. In contrast, if the problem under consideration involves processes controlled by interfacial area (for example the aggregation of crystals to make sintered clusters, or the adsorption of water onto the surface of soil particles) then $D_{3,2}$ would be the best measure of average particle dimension. This is because the amount of surface area $S$ per unit volume of sample is simply $S=6 \phi / D_{3,2}$, where $\phi$ is the volume fraction of spherical grains. If we are interested in the permeability of rocks then we note that the Kozeny-Carman relation (Carman, 1937) gives an approximate expression for the hydrodynamic permeability in terms of $S$, so that $D_{3,2}$ is once more a key quantity. Hydrodynamic permeability may also be relevant to sedimentation of concentrated suspensions, as the rate of sedimentation is likely to be determined by D'Arcy flow through the bed as a whole, rather than by particles settling individually. In Ostwald ripening, crystal growth is driven by interfacial energy, but in the scaling regime of LSW theory (Lifshitz and Soyolov, 1961; Wagner, 1961) all the mean diameters have the same cube root dependence on time, so there is no obvious preferred choice in this case. Comparison of several mean diameters can, however, be used to shed light on whether Ostwald ripening is the dominant growth mechanism. For settling of dilute suspensions, we show below that $D_{3,1}$ is the more relevant parameter to calculate. As a less geologically relevant aside, statistical studies suggest that when observers look at cross-sections of different sphere distributions, they tend to rank them by size according to $D_{2,2}$ (Alderliesten, 2008); a correlation which has yet to be given a rigorous (physiological) explanation.

In physical chemistry, where size distributions are often accessed by light-scattering techniques, the most commonly quoted mean diameters for polydisperse systems are $D_{4,3}$ and $D_{3,2}$ (McClements, 2016) and, unless there are specific phenomena under consideration that call for other measures, these two quantities provide a good first characterization of the size distribution. Gathering data for both also has the advantage that their ratio $D_{4,3} / D_{3,2}$ (which is never less than 1 , and only equal to 1 for monodisperse spheres) can be used to quantify the width of the size distribution. For example, for the specific case of a lognormal distribution in which the fraction of the number of spherical particles with diameters between $D$ and $D+\delta D$ is $F(D) \delta D$, where (Farr, 2013)

$$
F(D)=\frac{1}{D \sigma \sqrt{2 \pi}} \exp \left\{-\frac{\left[\ln \left(D / D^{*}\right)\right]^{2}}{2 \sigma^{2}}\right\}
$$

and $D^{*}=D_{4,3} \exp \left(-7 \sigma^{2} / 2\right)$, then the logarithmic width $\sigma$ (a simple measure of the range of particle sizes present in the rock) can be calculated from

$$
\exp \left(\sigma^{2}\right)=\frac{D_{4,3}}{D_{3,2}}
$$

\section{Sections through sphere distributions}

A thin section of a rock containing spherical or subspherical particles can be thought of as a plane section through a random distribution of spheres. If we measure all the $v_{\text {circ }}$ diameters $\left\{d_{i}\right\}$ of the circular cuts through the particles for $1 \leq i \leq v_{\text {circ }}$ in some region of the thin section (where lower case $d$ 's refer to two-dimensional sections and upper case $D$ 's to three-dimensional spheres), we can use these data to define the various mean circle diameters, in the same way as for the different mean sphere diameters. For example $d_{3,2}$ and $d_{2,1}$ 
would be

$$
\begin{aligned}
& d_{3,2} \equiv \sum_{i} d_{i}^{3} / \sum_{i} d_{i}^{2}, \\
& d_{2,1} \equiv \sum_{i} d_{i}^{2} / \sum_{i} d_{i}^{1} .
\end{aligned}
$$

Provided the number $v_{\text {circ }}$ of measured grains in the sample is very large, simple integration (see Appendix) shows that the mean sphere diameters can be obtained from different mean circle diameters thus:

$$
\begin{aligned}
D_{1,0} & =\left(\frac{\pi}{2}\right) d_{0,-1} \approx 1.571 d_{0,-1} \\
D_{2,1} & =\left(\frac{4}{\pi}\right) d_{1,0} \approx 1.273 d_{1,0} \\
D_{3,2} & =\left(\frac{3 \pi}{8}\right) d_{2,1} \approx 1.178 d_{2,1} \\
D_{4,3} & =\left(\frac{32}{9 \pi}\right) d_{3,2} \approx 1.132 d_{3,2}
\end{aligned}
$$

and the number, $N$, of spheres per unit volume can be deduced from the number, $n$, of circles per unit area through

$$
N=\frac{2 n}{\left(\pi d_{0,-1}\right)} \approx \frac{0.637 n}{d_{0,-1}}
$$

where $d_{0,-1}$ is the harmonic mean of the circle diameters (the reciprocal of the arithmetic mean of the reciprocals of the diameters).

Although these relations are independent of the size distribution of spheres, they are only exact in the limit of an infinite number of individual measurements. For a finite number of measurements of individual particles, there will be some scatter in results if different particle populations are measured (e.g. different parts of the same thin section, or different thin sections of the same sample), and there may also be some systematic error in the mean taken over many realizations of the experiment. As an extreme example of systematic bias, if only one grain is measured (i.e. if $v_{\text {circ }}=1$ ), all the different averages $d_{2,1}, d_{3,2}$ etc. would be identical, leading to the absurd prediction $D_{4,3}<D_{3,2}$, which can never be true for any sphere size distribution.

\section{Confidence intervals: how many circular sections to measure?}

In this section we address the question of how many particles should be measured to achieve a given level of confidence in the estimates for average grain diameter. The results of our analysis depend on the distribution of sizes and, for simplicity, we assume this to be lognormal. The different lognormal distributions we consider are shown in Fig. 1.

Suppose we measure the diameters of particles in a thin section, and then calculate estimates for $D_{4,3}$, $D_{3,2}$ and $D_{4,3} / D_{3,2}$ using equations 11 and 12 . If this procedure were performed several times, each time measuring a different population of particles in the thin section, the results would have some scatter, due to statistical fluctuations, which (together with any systematic bias) will give an estimate of the uncertainty in the result.

Figure 2 shows results where we have used a computer to generate monodisperse spheres in random
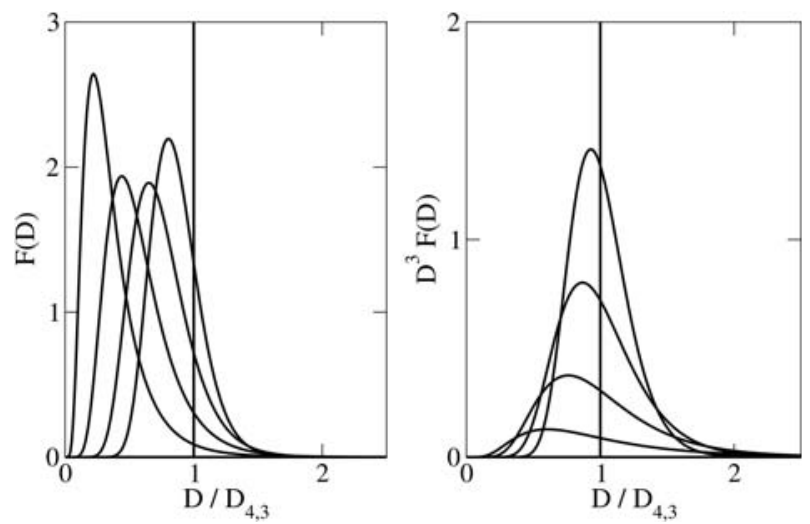

FIG. 1. Examples of lognormal distributions. Left hand plot shows the probability density function for the number of spheres [see equation 5], and right hand plot shows the same multiplied by the cube of the sphere diameter. Curves have (right to left) $D_{4,3} / D_{3,2}=1$ (the delta-function spike), 1.05, 1.1, 1.2 and 1.4. These values correspond to $\sigma=0,0.22$, $0.31,0.43$ and 0.58 , respectively. 
locations in space and generated circular sections from a plane drawn through this distribution. No account is taken of sphere overlaps, so the simulations strictly represent the dilute limit. The plots show the $68 \%$ confidence intervals for the predicted quantities compared to their true values (which are known in this case). The confidence intervals mean that $68 \%$ of the results lie in the interval; $68 \%$ being chosen because for a normal distribution this would represent plus or minus one standard deviation.

If we define the fractional error of the method to be the difference between the least accurate point in the confidence interval and the true value, divided by the true value, then we can plot this fractional error also for different lognormal sphere size distributions. This is done in Fig. 3, where we see that in general the fractional error is proportional to $v_{\text {circ }}^{-1 / 2}$, as would be expected from the central limit theorem, but overall it is harder to accurately measure parameters for the wider sphere size distributions.

For practical error estimation, we fit the various curves in Fig. 3 in the large $v_{\text {circ }}$ limit, and then use the $v_{\text {circ }}^{-1 / 2}$ dependence to extrapolate back to smaller sample sizes. The result is the following approximation for sphere size parameters, including an error estimate: First estimate the effective logarithmic width, $\sigma_{\text {est }}$, and fractional error, $e_{\text {est }}$ via:

$$
\begin{aligned}
& \sigma_{\text {est }}=\sqrt{\ln \left(\frac{0.961 d_{3,2}}{d_{2,1}}\right)} \\
& e_{\text {est }}=\frac{0.15}{\sqrt{\nu_{\text {circ }}}} \exp \left(5 \sigma_{\text {est }}\right),
\end{aligned}
$$

then the sphere statistics can be approximated via:

$$
\begin{aligned}
D_{4,3} & =1.132 d_{3,2}\left(1 \pm e_{\text {est }}\right) \\
D_{3,2} & =1.178 d_{2,1}\left(1 \pm e_{\text {est }}\right) \\
\frac{D_{4,3}}{D_{3,2}} & =0.961 \frac{d_{3,2}}{d_{2,1}}\left(1 \pm e_{\text {est }}\right) .
\end{aligned}
$$

\section{Sensitivity to ignoring the smallest circular cross sections}

The smallest cross-sections in a sample may fall below the limit of resolution in an image, so it is important to know how sensitive are the statistics we propose to the omission of the small tail of the circle distribution. Figure 4 shows similar data to Fig. 3, but where some of the small circles have been omitted from the statistics, specifically, all those circles with diameters less than some fraction $\alpha$ of $D_{4,3}$. We see that estimates of $D_{4,3}$ itself are not affected materially even when $\alpha$ is as large as 0.2 , while estimates for $D_{3,2}$ are a little more sensitive, and require $\alpha \leq 0.1$ for there to be no measurable effect. This lack of sensitivity, of both the volumeweighted and area-weighted averages, to an under-representation of the smallest particles is unsurprising. Therefore for the statistics we propose here $\left(D_{4,3}, D_{3,2}\right.$ and their ratio), a sensible rule of thumb would be to check post hoc that all circles larger than one fifth of the calculated value of $D_{4,3}$ have been included in the averages, and preferably all those larger than one tenth of $D_{4,3}$.
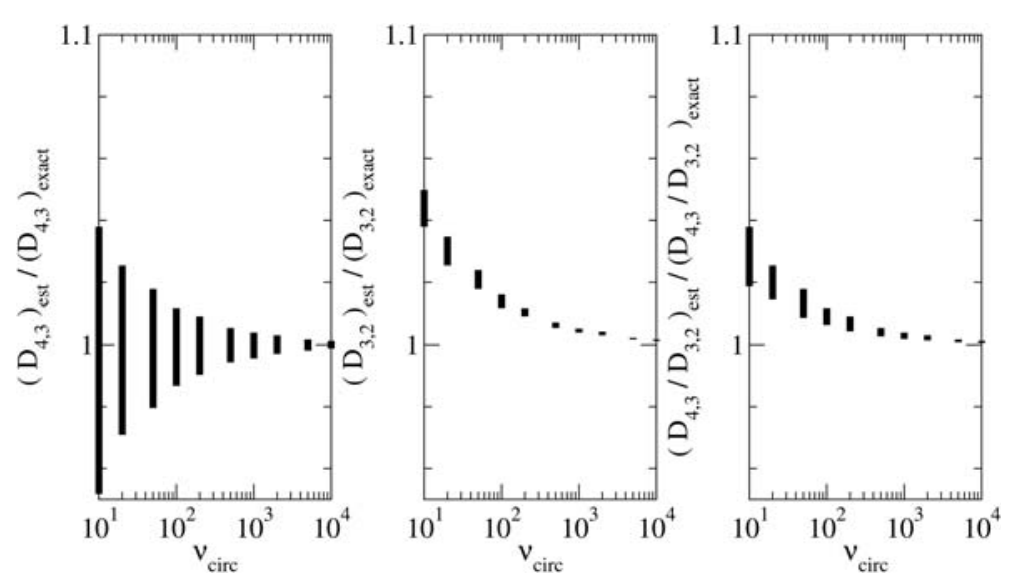

FIG. 2. Confidence intervals for predictions (estimates) of sphere size parameters from circular sections, compared to the known exact values. These data are for a computer generated random distribution of monodisperse spheres, and show that the estimates converge to the correct values as the number $v_{\text {circ }}$ of sampled circles increases. 

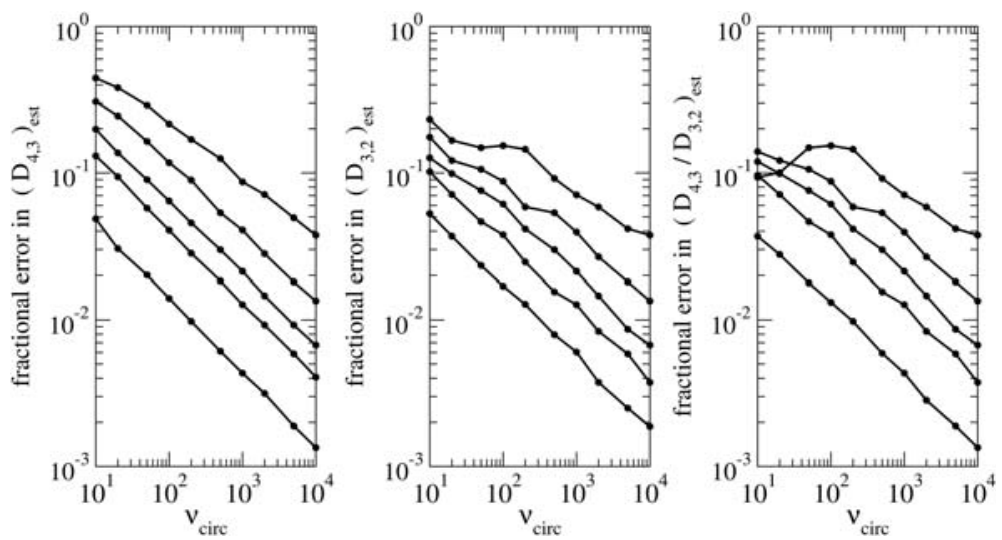

FIG. 3. Relative errors in predictions of sphere size parameters from circular sections. The data are from computer generated random distributions of lognormal sphere sizes, with log widths (bottom to top in each plot) $\sigma=0,0.22,0.31$, 0.43 and 0.58 (as for Fig. 1), which correspond to $D_{4,3} / D_{3,2}=1,1.05,1.1,1.2$ and 1.4, respectively. The estimates converge to the correct values as the number $v_{\text {circ }}$ of sampled circles increases.

\section{Does the procedure work for cubic crystals?}

For non-spherical particles, we define the equivalent diameter of a particle as the diameter of a sphere that has the same volume. Similarly for the cross-sections, we define the equivalent circle diameter as the diameter of a circle with the same area as the cross-section of the particle (which for a cubic particle will be a polygon with 3 to 6 sides; see Higgins (1994) and Morgan and Jerram (2006) for examples).

Figure 5 shows the errors incurred when the proposed procedure in this paper (derived for spheres) is applied to a random distribution of randomly oriented cubes. While the errors do not die out at $v_{\text {circ }} \rightarrow \infty$, and never fall below $\sim 3 \%$, this procedure can indeed be used to obtain reasonable estimates of the equivalent mean diameters for the cube population.

We note however that although values for equivalent $D_{4,3}, D_{3,2}$ and their ratio are well predicted, it would not be appropriate to apply the sphere result of $6 \phi / D_{3,2}$ to estimate the specific surface area in the system. The correct expression for cubes results in a surface area about $24 \%$ higher (the surface area of a cube being $24 \%$ higher than a sphere of the same volume). Instead, the standard stereological method (Russ, 1986) that the specific
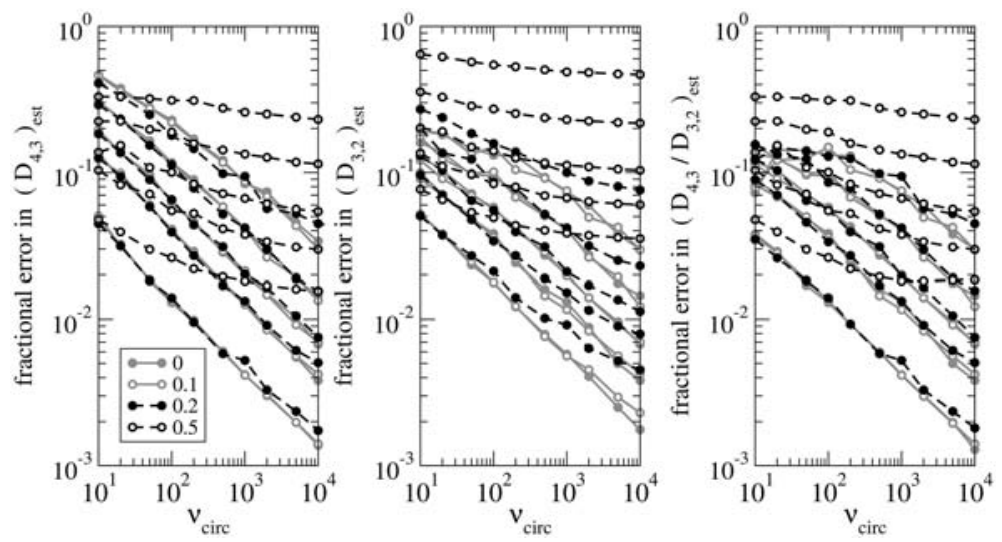

FIG. 4. Relative errors in predictions of sphere size parameters from circular sections. The data are from the same procedure as Fig. 3, but circles with diameters smaller than a fraction $\alpha$ of $D_{4,3}$ have been omitted from the statistics. The plots show the cases $\alpha=0$ (the same as Fig. 3), 0.1, 0.2 and 0.5 . 

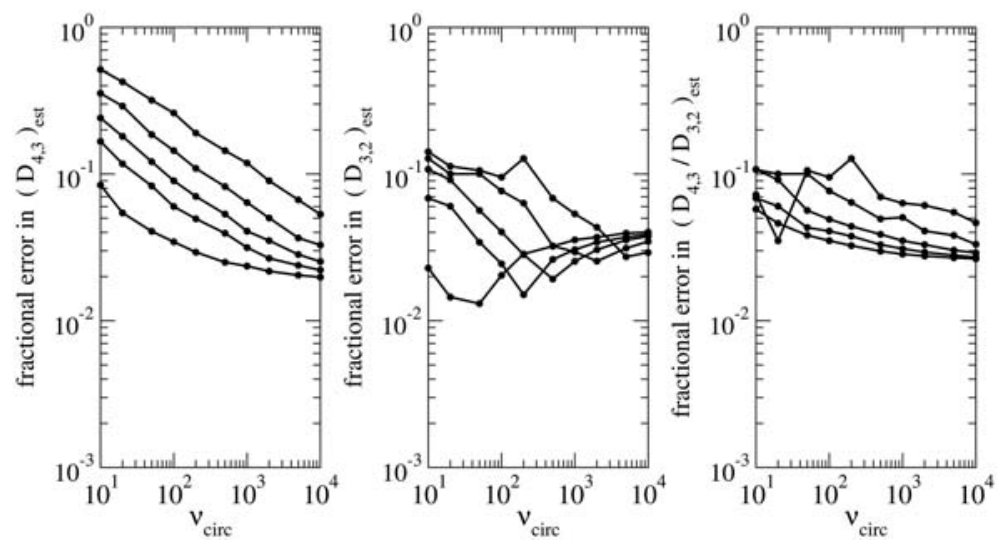

FIG. 5. Relative errors in predictions of cube size parameters for randomly positioned and randomly oriented cubes in space. The equivalent spheres and equivalent circles for polygonal cross sections are calculated, and the same procedure is used as in Fig. 3.

surface area is $(4 / \pi)$ times the perimeter per unit area in the cross section would be the appropriate method for this statistic.

\section{Application to gravitational settling of a polydisperse grain population}

An almost universal process occurring during the solidification of basaltic magma is the relative movement of crystals and residual liquid under the influence of gravity. It is this process which is the fundamental driver for fractionation. Here we discuss a simple treatment of settling under gravity of an initially dilute suspension of crystals entrained in a basaltic magma. If we assume that emplacement of the crystal-bearing magma is essentially instantaneous, and that the crystalbearing magma has a Newtonian rheology, the initial buildup rate $\dot{H}$ of the thickness of the layer of crystals on the magma chamber floor for a dilute suspension of polydisperse spheres in a nonconvecting Newtonian liquid can be calculated as follows:

Suppose there are $N$ spheres per unit volume in suspension and let the fraction of the number of these spheres that have diameters between $D$ and $\delta D$ be $F(D) \delta D$, so that $F(D)$ is a normalized probability density: $\int_{0}^{\infty} F(D) d D=1$. These spheres are initially randomly distributed in a liquid column above the floor, and we assume that their growth during settling is negligible. If all the spheres were of diameter $D$ then due to Stokes' law (the suspension is dilute) they would all settle at a speed

$$
u(D)=\frac{\mathrm{g} \Delta \rho}{18 \eta} D^{2},
$$

where $\eta$ is the fluid viscosity, $g$ the acceleration due to gravity and $\Delta \rho$ the difference in density between spheres and liquid. Thus, in unit time, the volume of spheres whose centres pass through a horizontal plane in the fluid per unit area of that plane will be

$$
\dot{V}_{s}=\frac{\pi D^{3}}{6} N u(D)=\frac{\pi \mathrm{g} \Delta \rho N}{108 \eta} D^{5},
$$

which represents the instantaneous buildup of sphere volume on the floor. For a range of sphere sizes, this rate will be

$\dot{V}_{s}=\frac{\pi \mathrm{g} \Delta \rho N}{108 \eta} \int_{0}^{\infty} D^{5} F(D) d D=\frac{\pi \mathrm{g} \Delta \rho N}{108 \eta} D_{5,0}^{5}$

The number $N$ of spheres per unit volume is related to the volume fraction $\phi_{\text {susp }}$ in suspension through $\phi_{\text {susp }}=\pi N D_{3,0}^{3} / 6$, while the rate of buildup of the layer thickness is related to $\dot{V}_{s}$ through $\dot{H}=\dot{V}_{S} / \phi_{\text {sed }}$, where $\phi_{\text {sed }}$ is the volume fraction of spheres in the sediment at the time they are deposited. Combining these with equation 20 and 2 we obtain $\dot{H}=\phi_{\text {susp }}$ g $\Delta \rho D_{5,3}^{2} /\left(18 \phi_{\text {sed }} \eta\right)$. However, the size distribution $F(D)$ applies to the spheres in suspension, while we only have access to the size distribution in the sediment. Suppose there are $N_{i \text {;susp }}$ spheres per unit volume in the suspension with sizes close to $D_{i}$. The number per unit volume $N_{i \text {;sed }}$ in the sediment will be increased in proportion to the speed of sedimentation. 
Therefore $D_{5,3}$ in the suspension (at this moment in time) will be equal to $D_{3,1}$ in the sediment, and so we obtain

$$
\dot{H}=\frac{\phi_{\text {susp }} \mathrm{g} \Delta \rho}{18 \phi_{\text {sed }} \eta} D_{3,1}^{2},
$$

where $D_{3,1}$ is now obtained from the distribution of sphere sizes in the sediment (at the time of deposition). Note that this is a local measure at this point in the accumulating sediment, and a more global analysis capturing variations with sediment depth could be performed, based on the conservation laws of sedimentation in partial differential form (Simakin et al., 1998).

As settling proceeds, the suspension becomes less dilute near the floor and the particles become closer together. During the final stages of sedimentation therefore, Stokes' Law no longer holds and sedimentation rates become controlled more by the permeability of the particle accumulation.

\section{Olivine settling in the Shiant Isles Main Sill}

To illustrate our treatment of settling timescales using average particle size we focus on the Shiant Isles Main Sill, which is the largest of the four separate Tertiary alkaline basalt sills exposed on the Shiant Isles (Outer Hebrides, Scotland), and intruded into Jurassic sediments (Gibb and Henderson, 1984). The Shiant Isles Main Sill is $165 \mathrm{~m}$ thick (Gibb and Henderson, 1984) and is a composite body (Drever and Johnston, 1959; Gibb and Henderson, 1989, 1996). The bulk of the sill
(135 $\mathrm{m}$ of stratigraphy) formed from a single pulse of olivine-phyric magma that contained $10 \mathrm{vol} . \%$ olivine phenocrysts together with 1-2 wt.\% Cr-spinel and a small amount of plagioclase (Gibb and Henderson, 2006). The olivine phenocrysts settled to the (contemporary) floor of the intrusion to form a picrodolerite $\sim 45 \mathrm{~m}$ thick, leaving an essentially aphyric magma that crystallized to form the remainder (a crinanite, dominated by plagioclase and augite, with interstitial olivine, Fe-Ti oxides and analcime). Here we concentrate on the olivine accumulation that forms the picrodolerite. The olivine grains are generally equant and rounded, commonly forming clusters and loose chains in which the individual grains are joined by small areas of grain boundary.

We selected sample SC459 which was collected from $29.72 \mathrm{~m}$ above the floor of the composite sill, $\sim 7 \mathrm{~m}$ from the base of the picrodolerite/crinanite unit and therefore within the lower part of the picrodolerite (Gibb and Henderson, 1996). Olivine grain sizes were determined using photomicrographs of a single thin section under planepolarized light. Outlines of individual grains were traced by hand from the photomicrographs, with direct comparison of the thin section under crossed polars to distinguish individual grains in clusters (Fig. 6). The areas of all grain intersections were determined using ImageJ software, and the diameters of circles with the same areas were then calculated. A total of 517 olivine crystals were identified. The statistics of the population are shown in Table 1. From these, using equations 14 and 15 we obtain $\sigma_{\text {est }}=0.59$ and $e_{\text {est }}=0.12$.
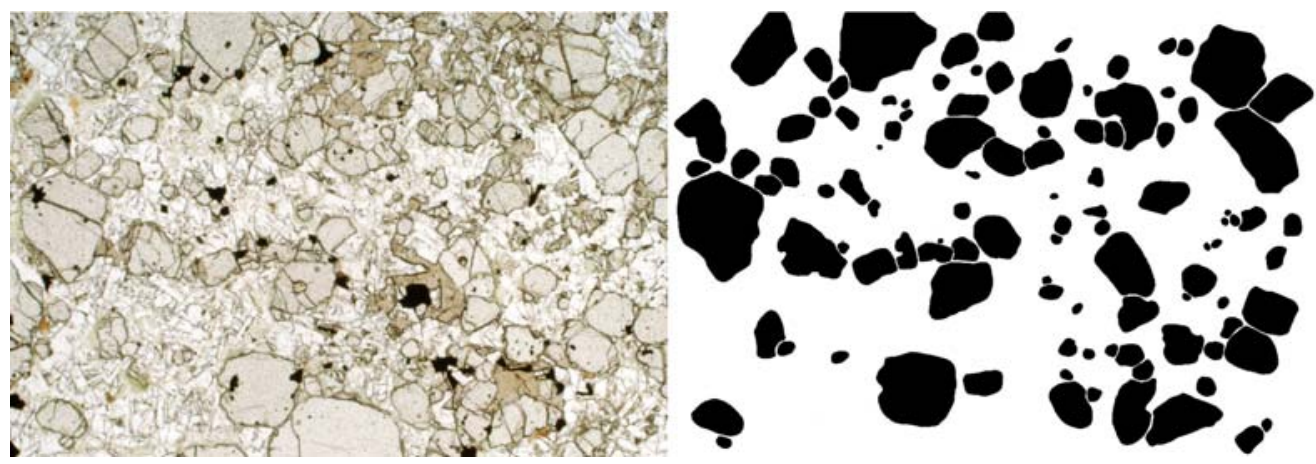

FIG. 6. Left: photomicrograph (plane-polarized light) of sample SC459 from the Shiant Isles Main Sill, containing loose clusters and chains of olivine phenocrysts set in a matrix of interstitial plagioclase and augite. Note the rare grains of chromite (opaque) that were also part of the crystal load of the incoming magma. Width of image is $4.5 \mathrm{~mm}$. Right: same image segmented by hand. We measured the area of each olivine crystal and determined the diameter of the circle with the same area. 
TABLE 1. Statistics for equivalent diameters $\left\{d_{i}\right\}$ of a total of $v_{\text {circ }}$ olivine grain crosssections from sample SC459 from the Shiant Isles Main Sill.

\begin{tabular}{ll}
\hline$v_{\text {circ }}=517$ & $\sum_{i}^{d} 3=14.13 \mathrm{~mm}^{3}$ \\
$d_{\min }=0.031 \mathrm{~mm}$ & $\sum_{i}^{d} 4=8.841 \mathrm{~mm}^{4}$ \\
$d_{\max }=1.258 \mathrm{~mm}$ & $d_{2,1}=0.313 \mathrm{~mm}$ \\
$\sum_{i}^{d}=98.05 \mathrm{~mm}$ & $d_{3,2}=0.460 \mathrm{~mm}$ \\
$\sum_{i}^{d} 2=30.37 \mathrm{~mm}^{2}$ & $d_{2,0}=0.242 \mathrm{~mm}$
\end{tabular}

The smallest and largest equivalent diameters in the sample are $d_{\min }$ and $d_{\max }$.

Equations 16 to 18 then give the following estimates:

$$
\begin{gathered}
D_{4,3}=0.53 \pm 0.06 \mathrm{~mm} \\
D_{3,2}=0.37 \pm 0.05 \mathrm{~mm} \\
\frac{D_{4,3}}{D_{3,2}}=1.41 \pm 0.18
\end{gathered}
$$

As $d_{\min } / D_{4,3}=0.06$, which is much smaller than one fifth, we can be confident that these estimates are not affected by any smaller grains which were not resolved. We also note from equations $\mathrm{A} 12$ and A10 (see Appendix) that $D_{3,1}^{2}=1.5 d_{2,0}^{2}$ so, assuming $D_{3,1}$ has the same relative error as $D_{4,3}$ and $D_{3,2}$,

$$
D_{3,1}=0.30 \pm 0.04 \mathrm{~mm} .
$$

If we assume that the only mineral accumulating on the sill floor was olivine, we can calculate the original volume fraction of settled grains if we also assume that the interstitial liquid crystallized as a mixture of interstitial plagioclase, augite and overgrowths on the existing olivine grains. This means that the observed grains are all somewhat larger than the original settled population, and so a correction must be made for this post-accumulation overgrowth.

Let us suppose that a collection of spherical crystals, initially at a volume fraction $\phi$, changes in volume fraction by a small amount $\delta \phi$ through all the crystals increasing their diameters by the same amount $\delta D$. Because $\phi=\pi D_{3,0}^{3} N / 6$, we see, by expansion, that, to leading order in $\delta \phi$

$$
\delta D=\frac{D_{3,2} \delta \phi}{3 \phi} .
$$

Substituting this small change in diameter (equal for all the spheres) into the definition of $D_{j, k}$ in equation 2 , and assuming $j \neq k$, gives the small change $\delta D_{j, k}$ in $D_{j, k}$ :

$\delta D_{j, k}=D_{j, k}\left[\frac{\left(j / D_{j, j-1}\right)-\left(k / D_{k, k-1}\right)}{j-k}\right] \frac{D_{3,2} \delta \phi}{3 \phi}$.

As olivine is ophitic in the overlying crinanite we can assume (following Gibb and Henderson, 2006) that the mineral modes in the crinanite are representative of those that crystallized from the interstitial liquid in the picrodolerite. The average olivine mode for the crinanite is 6 vol.\% (using the 20 data points between $69.68 \mathrm{~m}$ and $147 \mathrm{~m}$ stratigraphic height given by Henderson et al. (2000)). The proportion of Sample SC459 that is olivine is $48 \mathrm{vol} \%$, which suggests that if the volume fraction of olivine in an olivine-only sediment at the time of deposition were $\phi_{\text {sed }}$ then $0.48=\phi_{\text {sed }}+0.06\left(1-\phi_{\text {sed }}\right)$, so $\phi_{\text {sed }} \approx 0.45$ and the change in volume fraction to remove the overgrowth is thus $\delta \phi=-0.03$. From this and equations 28 and 26 we estimate that removing the overgrowth reduces the value of $D_{3,1}$, but only by the insignificant amount of $3 \mu \mathrm{m}$, so we can continue to use the original value of $D_{3,1}=0.30 \mathrm{~mm}$.

The corrected olivine mode in SC459 is lower than the 56-54 vol.\% expected for a random loose packing of cohesionless monodisperse spheres (Onoda and Liniger, 1990; Ciamarra and Coniglio, 2008; Zamponi, 2008; Farrell et al., 2010), and lower still than random loose packings achieved for polydisperse particles (Epstein and Young, 1962; Jerram et al., 2003), suggesting that olivine was not the only phase settling from the incoming magma. However, the efficiency of random loose packing is reduced for strongly cohesive particles, for which a stable distribution can be achieved at lower volume fractions (Dong et al., 2006; Yang et al., 2007). The presence of highly non-spherical, loose clustered chains of olivine will reduce this still further (Campbell et al., 1978; Jerram et al., 2003), with packings as low as 37 vol. $\%$ observed for settled accumulations of loose chains and clusters of olivine and magnetite (Campbell et al., 1978),. If we assume that the crystal pile was not densified by compaction, shear or shaking, it is therefore plausible that the accumulated olivine grains in SC459 preserve a randomly loose packed, mechanically stable framework of crystals and loose crystal clusters and chains with an overall solid fraction of $\sim 45$ vol.\%.

Olivine in the basal picrodolerite is $\mathrm{Fo}_{80}(\mathrm{Gibb}$ and Henderson, 1996), which has a density of $3.29 \mathrm{~g} / \mathrm{cm}^{3}$. Gibb and Henderson (2006) provide 
two suggested compositions for the parental magma that formed the $135 \mathrm{~m}$ picrodolerite/ crinanite unit. Assuming an intrusion temperature of $1200^{\circ} \mathrm{C}$ (Gibb and Henderson, 2006), a confining pressure of $0.5 \mathrm{kbar}$ and $\mathrm{a}_{2} \mathrm{O}$ content of $1 \mathrm{wt} . \%$, the two parental magmas have a density of $2.6 \mathrm{~g} / \mathrm{cm}^{3}$, calculated according to the method of Bottinga and Weill (1970), using volume and thermal expansion data from Lange and Carmichael (1987); Kress and Carmichael (1991). Therefore the density difference is $\Delta \rho=700 \mathrm{~kg} \mathrm{~m}^{-3}$. The largest uncertainty relates to the viscosity of the liquid phase through which sedimentation occurs. From Giordano et al. (2008) the two compositions lead to predicted viscosities of 3.9 and $8.5 \mathrm{~Pa}$. Using the above properties in equation 22 , we obtain two values for the rate of buildup of the sediment in the magma chamber:

$$
\dot{H}=7.7 \mu \mathrm{ms}^{-1} \text { or } \quad 3.5 \mu \mathrm{m} s^{-1} .
$$

The layer of sedimented olivine is $45 \mathrm{~m}$ thick (Gibb and Henderson, 1989, 1996) so, provided convection did not significantly delay the process, we predict a time of around 280 or 600 days for the layer to accumulate. This is much faster than the cooling time of the sill: a simple upper bound on the time for solidification can be obtained by assuming the entire thickness of the $165 \mathrm{~m}$ sill is intruded into cold country rock, which results in an estimate of 90 years from emplacement to complete crystallization (using the simple calculation developed by Holness et al., 2012).

Note that although the volume fraction of the settled olivine is $\sim 45 \mathrm{vol} \%$ in the lower part of the picrodolerite, the olivine mode decreases upwards towards the crinanite (Gibb and Henderson, 1996).
It is probable that this reduction is matched by an increase in the accumulation of another phase, such as plagioclase. The implications of this will be explored in a future contribution.

\section{Mechanisms of droplet growth in phase- separating magmas}

Silicate liquid immiscibility in basaltic systems, first recognized by Roedder and Weiblen (1971) (with significant further observations by Philpotts $(1979,1982))$, is recognized increasingly as an important factor controlling fractionation and the compositions of erupted magmas (Veksler et al., 2007; Charlier et al., 2011). The potential for immiscibility to affect the liquid line of descent on the scale of a magma chamber depends on the ease with which the two conjugate liquids can separate under the influence of gravity (e.g. Holness et al., 2011; VanTongeren and Mathez, 2012) and this, in turn, is affected strongly by the coarseness of the emulsion (e.g. Chung and Mungall, 2009). At present almost nothing is known about the kinetics of emulsion coarsening (e.g. Martin and Kushiro, 1991; Veksler et al., 2010). However the size distribution of the droplets potentially carries information about the mechanisms of their formation and subsequent growth.

One possible scenario for emulsion formation is that there is an initial interval when many nuclei form, followed by evolution of the structure without further nucleation. This could happen, for example, with spinodal decomposition, or if the system is between the binodal and spinodal lines and there are many potential sites for heterogeneous nucleation. Once droplets have formed in such a system,

TABLE 2. Statistics on emulsion droplet size distribution in immiscible basaltic glasses from the experimental charges of Charlier and Grove (2012).

\begin{tabular}{lrccccc}
\hline Sample & $v_{\text {drop }}$ & Final $T\left({ }^{\circ} \mathrm{C}\right)$ & Equilibration time (hours) & $\mathrm{D}_{4,3} / \mu \mathrm{m}$ & $\mathrm{D}_{3,2} / \mu \mathrm{m}$ & $\mathrm{D}_{4,3} / \mathrm{D}_{3,2}$ \\
\hline SI-5 & 282 & 1006 & 96 & $3.94 \pm 1.54$ & $2.22 \pm 0.87$ & $1.77 \pm 0.69$ \\
SI-8 & 484 & 963 & 48 & $2.26 \pm 0.30$ & $1.59 \pm 0.21$ & $1.42 \pm 0.19$ \\
SI-13 & 299 & 1020 & 64 & $1.36 \pm 0.04$ & $1.28 \pm 0.04$ & $1.06 \pm 0.03$ \\
M-5 & 3205 & 1005 & 92 & $2.42 \pm 0.10$ & $1.80 \pm 0.07$ & $1.35 \pm 0.05$ \\
M-6 & 621 & 963 & 48 & $1.85 \pm 0.11$ & $1.51 \pm 0.09$ & $1.23 \pm 0.07$ \\
M-9 & 2017 & 1020 & 64 & $2.22 \pm 0.09$ & $1.75 \pm 0.07$ & $1.27 \pm 0.05$
\end{tabular}

The number of droplets measured is $v_{\text {drop }}$. The equilibration time gives the time for which the charge was held at the final temperature after having been cooled from a starting temperature of $1100^{\circ} \mathrm{C}$ at $1{ }^{\circ} \mathrm{C} / \mathrm{hr}$. Charges SI-5, SI- 8 and SI- 13 have a bulk composition identical to that of a dyke cutting the Sept Iles intrusion and charges M-5, M-6 and M-9 have a bulk composition typical of an intermediate basalt from the Mull Tertiary volcano (for further details see Charlier and Grove (2012)). 


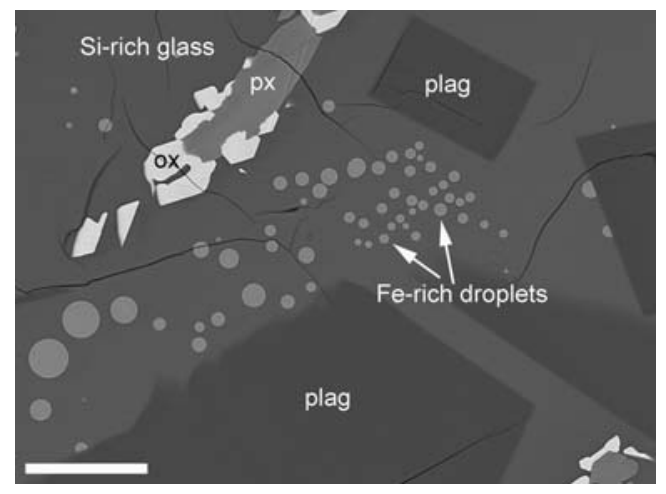

FIG. 7. Back-scatter electron image of experimental charge SI-5 (see text for details), showing plagioclase (plag), pyroxene (px) and oxide grains (ox) set in a silica-rich glass containing droplets of an immiscible Fe-rich liquid (now quenched to glass). Most of the droplets appear to have nucleated homogeneously, but several droplets attached to the plagioclase grain at the far right probably nucleated heterogeneously on the plagioclase surface. The scale bar is $20 \mu \mathrm{m}$ long.

they can grow by various mechanisms. In a non-convecting liquid, growth can happen either by Ostwald ripening, or through coalescence as droplets diffuse and collide under Brownian forces. Both of these mechanisms lead to a state where all the mean diameters grow as the cube root of time (Crist and Nesarikar, 1995), but each has a characteristic size distribution, which can be probed by moment-based averages. From the derivations in Appendix B, we see that Ostwald ripening leads asymptotically to $D_{4,3} / D_{3,2}=1.0239$, whereas the size distribution resulting from coalescence under Brownian forces is characterized by $D_{4,3} / D_{3,2}=1.0748$.

We analysed the sizes of droplets in experimental charges described previously by Charlier and Grove (2012). The charges, containing material representative of compositions of tholeiitic basalts from the Sept Iles intrusion (charges SI-5, SI-8 and SI-13, Table 2) and Mull (charges M-5, M-6 and M-9, Table 2), were cooled at $1^{\circ} \mathrm{C}$ per hour from a starting temperature of $1100^{\circ} \mathrm{C}$ to a range of temperatures at which they were held for periods of up to 96 hours before quenching. At the end of each experiment the charges contained solid phases (detailed in Table 2 of Charlier and Grove (2012)) together with Si-rich glass containing exsolved quenched droplets of Fe-rich liquid (Fig. 7). Using back-scatter images we measured the diameters of isolated droplets in large regions of glass, avoiding those in direct contact with the mineral phases (thus avoiding droplets that may have nucleated heterogeneously on the mineral surfaces).

We find $D_{4,3} / D_{3,2}$ values of between 1.06 and 1.8 , implying a size distribution in all cases except one (and including the estimated uncertainty) significantly more broad than either of these two mechanisms would predict. We interpret this discrepancy as evidence for continuous nucleation while the existing droplets are ripening, consistent with the design of the experiments in which the temperature was reduced at a steady rate into the binodal.

\section{Conclusions}

Moment-based methods for particle size characterization provide a simple way to describe a population of (sub-) spherical particles (crystals, sedimentary clasts, emulsion droplets or bubbles), and have the advantage that exact results allow the different averages of the three dimensional population to be deduced (with estimated error bars) from two dimensional sections. Which of the mean diameters to use depends on the phenomena of interest, but we suggest that $D_{4,3}$ and $D_{3,2}$ give a good first characterization of the population (including the spread of diameters), while $D_{3,1}$ can be useful for accumulations of sedimenting grains or rising bubbles. Their ratio is a measure of the width of the size distribution, and this can carry information about growth mechanisms of inclusions.

\section{Acknowledgements}

We thank Fergus Gibb and Michael Henderson for their help to access material from the Shiant Isles Main Sill. We acknowledge the loan of Shiant samples from the British Geological Survey and thank Michael Togher for his helpfulness and efficiency at facilitating the loans. We are grateful to Bernard Charlier for providing images of his experimental charges, and for the loan of the charges themselves to enable us to create further images. Comments from two anonymous reviewers greatly improved the manuscript. V.C.H. is supported by a Natural Environment Research Council studentship. M.B.H. acknowledges support from the Natural Environment Research Council [grant number NE/J021520/1].

\section{References}

Alderliesten, M. (1990) Mean particle diameters, Part I, Evaluation of definition systems. Particle Particle Systems Characterization, 7, 233-241. 
Alderliesten, M. (2008) Mean particle diameters: from statistical definition to physical understanding. Unpublished $\mathrm{PhD}$ thesis, TU Delft, The Netherlands.

Bottinga, Y. and Weill, D.F. (1970) Densities of liquid silicate systems calculated from partial molar volumes of oxide components. American Journal of Science, 269, 169-182.

Cabane, H., Laporte, D. and Provost, A. (2001) Experimental investigation of the kinetics of Ostwald ripening of quartz in silicic melts. Contributions to Mineralogy and Petrology, 142, 361-373.

Cabane, H., Laporte, D. and Provost, A. (2005) An experimental study of Ostwald ripening of olivine and plagioclase in silicate melts: implications for the growth and size of crystals in magmas. Contributions to Mineralogy and Petrology, 150, 37-53.

Campbell, I.H., Roeder, P.L. and Dixon, J.M. (1978) Plagioclase buoyancy in basaltic liquids as determined with a centrifuge furnace. Contributions to Mineralogy and Petrology, 67, 369-377.

Carlson, W.D. and Denison, C. (1992) Mechanisms of porphyroblast crystallisation: results from high-resolution computed X-ray tomography. Science, 257, 1236-1239.

Carman, P.C. (1937) Fluid flow through granular beds. Transactions, Institution of Chemical Engineers, London, 15, 150-166.

Cashman, K.V. (1992) Groundmass crystallisation of Mount St. Helens dacite, 1980-1986: a tool for interpreting shallow magmatic processes. Contributions to Mineralogy and Petrology, 109, 431-449.

Cashman, K.V. (1993) Relationship between plagioclase crystallisation and cooling rate in basaltic melts. Contributions to Mineralogy and Petrology, 113, 126-142.

Cashman, K.V. and Marsh, B.D. (1988) Crystal size distribution (CSD) in rocks and the kinetics and dynamics of crystallization II: Makaopuhi lava lake. Contributions to Mineralogy and Petrology, 99, 292-305.

Charlier, B. and Grove, T.L. (2012) Experiments on liquid immiscibility along tholeiitic liquid lines of descent. Contributions to Mineralogy and Petrology, 164, 27-44.

Charlier, B., Namur, O., Toplis, M.J., Schiano, P., Cluzel, N., Higgins, M.D. and Vander Auwera, J. (2011) Large-scale silicate liquid immiscibility during differentiation of tholeiitic basalt to granite and the origin of the Daly gap. Geology, 39, 907-910.

Chung, H.-Y. and Mungall, J.E. (2009) Physical constraints on the migration of immiscible fluids through partially molten silicate, with special reference to magmatic sulphide ores. Earth and Planetary Science Letters, 286, 14-22.

Ciamarra, M.P. and Coniglio, A. (2008) Random very loose packings. Physical Review Letters, 101, 128001.
Crist, B. and Nesarikar, A.R. (1995) Coarsening in polyethylene-copolymer blends. Macromolecules, 28, 890-896.

Denison, C. and Carlson, W.D. (1997) Three-dimensional quantitative textural analysis of metamorphic rocks using high-resolution computed X-ray tomography: Part II. Application to natural samples. Journal of Metamorphic Geology, 15, 45-57.

Dong, K.J., Yang, R.Y., Zou, R.P. and Yu, A.B. (2006) Role of interparticle forces in the formation of random loose packing. Physical Review Letters, 96, 145505.

Drever, H.I. and Johnston, R. (1959) The lower margin of the Shiant Isles sill. Quarterly Journal of the Geological Society of London, 94, 343-365.

Duchêne, S., Pupier, E., Le Carlier De Veslud, C. and Toplis, M.J. (2008) A 3D reconstruction of plagioclase crystals in a synthetic basalt. American Mineralogist, 93, 893-901.

Epstein, N. and Young, M.J. (1962) Random loose packing of binary mixtures of spheres. Nature, 196, 885-886.

Farr, R.S. (2013) Random close packing fractions of lognormal distributions of hard spheres. Powder Technology, 245, 28-34.

Farrell, G.R., Martiniti, K.M. and Menon, N. (2010) Loose packings of frictional spheres. Soft Matter, 6, 2925-2930.

Gibb, F.G.F. and Henderson, C.M.B. (1984) The structure of the Shiant Isles sill complex, Outer Hebrides. Scottish Journal of Geology, 20, 21-29.

Gibb, F.G.F. and Henderson, C.M.B. (1989) Discontinuities between picritic and crinanitic units in the Shiant Isles sill: evidence of multiple intrusion. Geological Magazine, 126, 127-137.

Gibb, F.G.F. and Henderson, C.M.B. (1996) The Shiant Isles main sill: structure and mineral fractionation trends. Mineralogical Magazine, 60, 67-97.

Gibb, F.G.F. and Henderson, C.M.B. (2006) Chemistry of the Shiant Isles main sill, NW Scotland, and wider implications for the petrogenesis of mafic sills. Journal of Petrology, 47, 191-230.

Giordano, D., Russell, J.K. and Dingwall, D.B. (2008) Viscosity of magmatic liquids: a model. Earth and Planetary Science Letters, 271, 123-134.

Hammer, J.E., Cashman, K.V., Hoblitt, R.P. and Newman, S. (1999) Degassing and microlite crystallisation during pre-climatic events of the 1991 eruption of Mt. Pinatubo, Philippines. Bulletin of Volcanology, 60, 355-380.

Henderson, C.M.B., Gibb, F.G.F. and Foland, K.A. (2000) Mineral fractionation and pre- and post-emplacement processes in the uppermost part of the Shiant Isles main sill. Mineralogical Magazine, 64, 779-790.

Herd, R.A. and Pinkerton, H. (1997) Bubble coalescence in basaltic lava: Its impact on the evolution of bubble populations. Journal of Volcanology and Geothermal Research, 75, 137-157. 
Higgins, M.D. (1994) Numerical modelling of crystal shapes in thin sections: estimation of crystal habit and true size. American Mineralogist, 79, 113-119.

Higgins, M.D. (1996) Magma dynamics beneath Kameni volcano, Thera, Greece, as revealed by crystal size and shape measurements. Journal of Volcanology and Geothermal Research, 70, 37-48.

Higgins, M.D. (2000) Measurement of crystal size distributions. American Mineralogist, 85, 1105-1116.

Holness, M.B. (1997) Geochemical self-organisation of olivine-grade contact metamorphosed chert nodules in dolomite marble, Kilchrist, Skye. Journal of Metamorphic Geology, 15, 765-775.

Holness, M.B., Stripp, G., Humphrys, M.C.S., Veksler, I.V., Nielsen, T.F.D. and Tegner, C. (2011) Silicate liquid immiscibility within the crystal mush: late-stage magmatic microstructures in the Skaergaard Intrusion, East Greenland. Journal of Petrology, 52, 175-222.

Holness, M.B., Richardson, C. and Helz, R.T. (2012) Disequilibrium dihedral angles in dolerite sills: A new proxy for cooling rate. Geology, 40, 795-798.

Hughes, D.W. (1978) A disaggregation and thin section analysis of the size and mass distribution of the chondrules in the Bjurbole and Chainpur meteorites. Earth and Planetary Science Letters, 38, 391-400.

Jerram, D.A., Cheadle, M.J. and Philpotts, A.R. (2003) Quantifying the building blocks of igneous rocks: are clustered crystal frameworks the foundation? Journal of Petrology, 44, 2033-2051.

Johnson, M.R. (1994) Thin section grain size analysis revisited. Sedimentology, 41, 985-999.

Kong, M., Bhattacharya, R.N., Jama, C. and Basu, A. (1995) A statistical approach to estimate the 3D size distribution of spheres from 2D size distributions. Geological Society of America Bulletin, 117, 244-249.

Kress, V.C. and Carmichael, I.S.E. (1991) The compressibility of silicate liquids containing $\mathrm{Fe}_{2} \mathrm{O}_{3}$ and the effect of composition, temperature, oxygen fugacity and pressure on their redox states. Contributions to Mineralogy and Petrology, 108, 82-92.

Lange, R.A. and Carmichael, I.S.E. (1987) Densities of $\mathrm{Na}_{2} \mathrm{O}-\mathrm{K}_{2} \mathrm{O}-\mathrm{MgO}-\mathrm{FeO}-\mathrm{Fe}_{2} \mathrm{O}_{3}-\mathrm{Al}_{2} \mathrm{O}_{3}-\mathrm{TiO}_{2}-\mathrm{SiO}_{2}$ liquids: New measurements and derived partial molar properties. Geochimica et Cosmochimica Acta, 51, 2931-2946.

Lifshitz, I.M. and Slyozov, V.V. (1961) The kinetics of precipitation from supersaturated solid solutions. Journal of Physics and Chemistry of Solids, 19, 35-50.

Marsh, B.D. (1988) Crystal size distribution (CSD) in rocks and the kinetics and dynamics of crystallisation I. Theory. Contributions to Mineralogy and Petrology, 99, 277-291.

Martin, B. and Kushiro, Y. (1991) Immiscibility synthesis as an indicator of cooling rates in basalts. Journal of Volcanology and Geothermal Research, 45, 289-310.
McClements, D.J. (2016) Food emulsions, principles, practices and techniques. CRC Press, Taylor Francis Group.

Meinders, M.B.J., Kloek, W. and van Vliet, T. (2001) Effect of surface elasticity on Ostwald ripening in emulsions. Langmuir, 17, 3923-3929.

Mock, A. and Jerram, D.A. (2005) Crystal size distributions (CSD) in three dimensions: insights from the 3D reconstruction of a highly porphyritic rhyolite. Journal of Petrology, 46, 1525-1541.

Morgan, D.J. and Jerram, D.A. (2006) On estimating crystal shape for crystal size distribution analysis. Journal of Volcanology and Geothermal Research, 154, 1-7.

Onoda, G.Y. and Liniger, E.G. (1990) Random loose packings of uniform spheres and the dilatancy onset. Physical Review Letters, 64, 2727-2780.

Philpotts, A.R. (1979) Sillicate liquid immiscibility in tholeiitic basalts. Journal of Petrology, 20, 99-118.

Philpotts, A.R. (1982) Compositions of immiscible liquids in volcanic rocks. Contributions to Mineralogy and Petrology, 80, 201-218.

Robertson, J. and Barnes, S. (2015) Dynamics of magmatic sulphide droplets during transport in silicate melts and implications for magmatic sulphide ore formation. Journal of Petrology, 56, 2445-2472.

Roedder, E. and Weiblen, P.W. (1971) Petrology of silicate melt inclusions, Apollo 11 and Apollo 12 and terrestrial equivalents. Proceedings of the Second Lunar Science Conference, 1, 507-528.

Russ, J.C. (1986) Practical Stereology. Plenum Press, New York.

Sahagian, D.L. and Proussevitch, A.A. (1998) 3D particle size distributions from 2D observations: stereology for natural applications. Journal of Volcanology and Geothermal Research, 84, 173-196.

Simakin, A.G., Trubitsyn, V.P. and Kharybin, E.V. (1998) The size and depth distribution of crystal settling in solidifying magma chamber. Izvestiya, Physics of the Solid Earth, 34(8), 30-37.

Swift, D.L. and Friedlander, S.K. (1964) The coagulation of hydrosols by Brownian motion and laminar shear flow. Journal of Colloid Science, 19, 621-647.

VanTongeren, J.A. and Mathez, E.A. (2012) Large-scale liquid immiscibility at the top of the Bushveld Complex, South Africa. Geology, 40, 491-494.

Veksler, I.V., Dorfman, A.M., Borisov, A.A., Wirth, R. and Dingwell, D.B. (2007) Liquid immiscibility and the evolution of basaltic magma. Journal of Petrology, 48, 2187-2210.

Veksler, I.V., Kähn, J., Franz, G. and Dingwell, D.B. (2010) Interfacial tension between immiscible liquids in the system $\mathrm{K}_{2} \mathrm{O}-\mathrm{FeO}-\mathrm{Fe}_{2} \mathrm{O}_{3}-\mathrm{Al}_{2} \mathrm{O}_{3}-\mathrm{SiO}_{2}$ and implications for the kinetics of silicate melt unmixing. American Mineralogist, 95, 1679-1685. 
Wagner, C. (1961) Theorie der Alterung von Niederschlägen durch Umlosen (Ostwald-Reifung). Zeitschrift fur Elektrochemie, 65, 581-591.

Wicksell, S.D. (1925) The corpuscle problem, a mathematical study of a biometric problem. Biometrika, 17, 84-99.
Yang, R.Y., Zou, R.P., Dong, K.J., An, X.Z. and Yu, A. (2007) Simulation of the packing of cohesive particles. Computer Physics Communications, 177, 206-209.

Zamponi, F. (2008) Mathematical physics: packings close and loose. Nature, 453, 606-607.

\section{Appendix A: Solution for the moments}

In what follows, we shall use upper case symbols to refer to three-dimensional quantities, and lower case to refer to two-dimensional quantities. Suppose we have a random distribution of spheres in space, where there are $N$ spheres per unit volume. Further, suppose that the fraction of the number of spheres that have diameters in the range $D$ to $D+\delta D$ is $F(D) \delta D$, so that the probability density function $F(D)$ is normalized: $\int_{0}^{\infty} F(D) d D=1$. We then imagine passing a plane section through this distribution, which produces an infinite collection of circular cross-sections. Let there be $n$ such crosssections per unit area of the plane, and let the fraction of the number of circles which have diameters between $d$ and $d+\delta d$ be $f(d) \delta d$, so that $f(d)$ is also normalized: $\int_{0}^{\infty} f(d) d d=1$.

We define moments of these probability distributions in three and two dimensions, via

$$
\begin{gathered}
M_{k} \equiv \int_{0}^{\infty} D^{k} F(D) d D, \\
m_{k} \equiv \int_{0}^{\infty} d^{k} f(d) d d,
\end{gathered}
$$

and we see from equation 2 that the various average diameters ( $\operatorname{provided} j \neq k$ ) can be obtained from the moments:

$$
\begin{aligned}
D_{j, k} & \equiv\left(\frac{M_{j}}{M_{k}}\right)^{1 /(j-k)}, \\
d_{j, k} & \equiv\left(\frac{m_{j}}{m_{k}}\right)^{1 /(j-k)} .
\end{aligned}
$$

Imagine temporarily that the spheres were monodisperse, all having the same diameter $D_{0}$, so that

$$
F(D)=\hat{\delta}\left(D-D_{0}\right),
$$

where $\hat{\delta}(x)$ is the Dirac delta-function.

A sphere will only intersect the plane if it lies in a volume close to the plane, in particular if the perpendicular distance $y$ of the sphere centre from the plane is such that $|y|<D_{0} / 2$. Thus the number of circles per unit area will be $n=D_{0} N$.
Provided $|y|<D_{0} / 2$, the diameter of the circular section will be $d=\sqrt{D^{2}-4 y^{2}}$, so as $y$ is distributed uniformly, the probability density of circle diameters will be proportional to $(d d / d y)^{-1}$. Taking into account the normalization and $n=D_{0} N$, we obtain the distribution of the number of circles per unit area with different diameters for the monodisperse spheres case:

$$
\begin{aligned}
n f(d) & =\frac{N d}{\sqrt{D_{0}^{2}-d^{2}}} \text { for } D_{0}>d \\
& =\int_{d}^{\infty} \frac{N d}{\sqrt{D^{2}-d^{2}}} \hat{\delta}\left(D-D_{0}\right) d D .
\end{aligned}
$$

Equation A6 then generalizes immediately to the case where the spheres have a distribution of sizes, as the numbers of circles per unit area add up in a linear manner:

$$
n f(d)=\int_{d}^{\infty} \frac{N d}{\sqrt{D^{2}-d^{2}}} F(D) d D .
$$

We can now simply write down the moments of the two dimensional distribution according to equation A2 with the only 'trick' required being to reverse the order of integration (see Fig. 8) between lines 2 and 3 below:

$$
\begin{aligned}
m_{k} & \equiv \int_{0}^{\infty} d^{k} f(d) d d \\
& =\int_{0}^{\infty}\left\{\int_{d}^{\infty} \frac{N d^{k+1}}{n \sqrt{D^{2}-d^{2}}} F(D) d D\right\} d d \\
& =\int_{0}^{\infty}\left\{\int_{0}^{D} \frac{N d^{k+1}}{n \sqrt{D^{2}-d^{2}}} F(D) d d\right\} d D \\
& =\left(\frac{N}{n}\right)\left[\int_{0}^{\pi / 2}(\sin \theta)^{k+1} d \theta\right] M_{k+1} .
\end{aligned}
$$



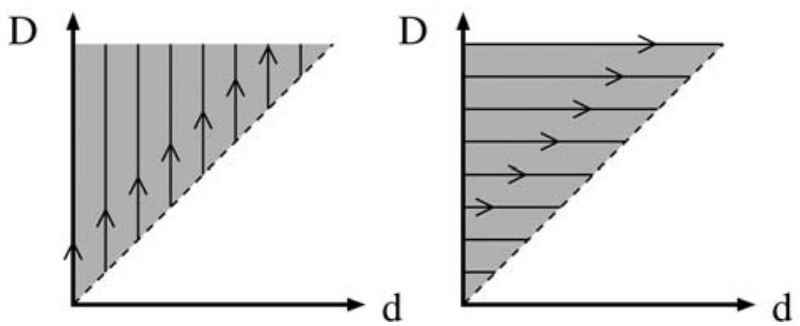

FIG. 8. Schematic of change of integration order in equation A8.

The relations below follow immediately:

$$
\begin{aligned}
M_{0} & =\left(\frac{n}{N}\right) \frac{2 m_{-1}}{\pi} \\
M_{1} & =\left(\frac{n}{N}\right) m_{0} \\
M_{2} & =\left(\frac{n}{N}\right) \frac{4 m_{1}}{\pi} \\
M_{3} & =\left(\frac{n}{N}\right) \frac{3 m_{2}}{2} \\
M_{4} & =\left(\frac{n}{N}\right) \frac{16 m_{3}}{(3 \pi)} \\
M_{5} & =\left(\frac{n}{N}\right) \frac{15 m_{4}}{8},
\end{aligned}
$$

and from these the relations between the different sphere and circle average diameters [equations 9 to 12] follow by means of equations $\mathrm{A} 3$ and $\mathrm{A} 4$.

We also note that by definition $M_{0}=m_{0}=1$, so dividing equation $\mathrm{A} 9$ by $m_{0}$, one obtains an equation relating the number of spheres per unit volume to the number of circles per unit area in the cross section:

$$
N=\frac{2 n m_{-1}}{\pi m_{0}}=\frac{2 n}{\pi d_{0,-1}} .
$$

\section{Appendix B: Droplet size distributions for different growth mechanisms}

In the LSW theory of Ostwald ripening (Lifshitz and Soyolov, 1961; Wagner, 1961), the size distribution of droplets eventually achieves a selfsimilar form in which there is a critical radius which grows as the cube root of time. If $u$ is the ratio of the droplet radius to this critical radius, the normalized number density of droplets is given by

$$
F(u)=\frac{81 e u^{2} \exp (1 /(2 u / 3-1))}{\sqrt[3]{32}(u+3)^{7 / 3}(1.5-u)^{11 / 3}}
$$

where $0<u<1.5$ and zero otherwise (Meinders et al., 2001). This distribution can be integrated numerically to obtain moments of the distribution, and specifically we find

$$
\frac{D_{4,3}}{D_{3,2}} \equiv \frac{\int u^{4} F(u) d u \int u^{2} F(u) d u}{\left(\int u^{3} F(u) d u\right)^{2}}=1.0239
$$

Alternatively, droplets can grow through coalescence as they diffuse under Brownian motion. If we accept Smoluchowski's approximation to Brownian aggregation (Swift and Friedlander,
1964) then, starting with monodisperse droplets of number density $n_{1}(0)$ at time $t=0$, there will be a distribution of sizes at a later time $t$. Specifically, let $n_{k}(t)$ be the number of droplets per unit volume that are $k$ times the volume of the initial droplets, then Smoluchowski's approximation gives

$$
n_{k}(t)=n_{1}(0) \frac{(t / \tau)^{k-1}}{(1+(t / \tau))^{k+1}}
$$

where $\tau \equiv 3 \eta /\left(4 k_{\mathrm{B}} T n_{1}(0)\right)$ and $k_{\mathrm{B}}$ is Boltzmann's constant, $T$ the temperature and $\eta$ the viscosity of the continuous phase. In the limit of large time and large $k$, this reduces to

$$
n_{k}(t) \approx n_{1}(0)\left(\frac{t}{\tau}\right)^{-2} \exp \left(\frac{-k \tau}{t}\right)
$$

Now, if the initial droplets have a volume $v_{1}$ then the diameter of a droplet formed from $k$ of these is $D(k)=\left(6 v_{1} k / \pi\right)^{1 / 3}$. Thus the number of droplets per unit volume with diameters in the range $D$ to 
ROBERT S. FARR ETAL.

$D+\delta D$ is $F(D) \delta D$ where

$$
\begin{aligned}
F(D) & =n_{k}(t)\left(\frac{d D(k)}{d k}\right)^{-1} \\
& =\frac{\pi D^{2} n_{1}(0)}{2 v_{1}(t / \tau)^{2}} \exp \left(-\frac{\pi D^{3} \tau}{6 v_{1} t}\right) .
\end{aligned}
$$

From this we find

$$
\frac{D_{4,3}}{D_{3,2}}=1.0748
$$

